\title{
XLVIII. On the conversion of right ascension and declination into longitude and latitude, and vice versâ
}

\section{Prof. Encke}

To cite this article: Prof. Encke (1830) XLVIII. On the conversion of right ascension and declination into longitude and latitude, and vice versâ , Philosophical Magazine Series 2, 7:41, 330-334, DOI: $10.1080 / 14786443008675303$

To link to this article: http://dx.doi.org/10.1080/14786443008675303

曲 Published online: 14 Jul 2009.

Submit your article to this journal $[\pi$

ЏII Article views: 3

Q View related articles $\longleftarrow$ 
$r$ and $t$, or the forms of the arbitrary functions in the complete integral, (which in this instance may be obtained,) be determined on the supposition that the origin and direction of coordinates are not fixed. I must, however, observe that the problem, selected in illustration of these principles; viz. to find the velocity at the vena contracta of a stream issuing from a small orifice in any vessel, is incorrectly solved. An accurate solution of this problem, and a fuller consideration of the whole subject, will be found in the memoir above alluded to.

Trin. Coll. Cambridge,

March 15, 1830.

XLVIII. On the Conversion of Right Ascension and Declination into Longitude and Latitude, and vice versâ. By Prof. ENCKE*.

VARIOUS applications have been made to me, requesting that I would give in the Ephemeris, besides the geocentric right ascensions and declinations of the planets, also their latitudes and longitudes, as was usual in the former construction of the Epherneris, it having been hitherto the custom to express the places of the planets by these latter co-ordinates. Without considerably enlarging the book, this wish could not be fulfilled; I have, however, endeavoured to supply this want, as far as it may still exist, by the following tables for approximately finding these quantities with an accuracy sufficient for all useful purposes. If only minutes of longitude and latitude are required for heavenly bodies moving in the zodiac, neither trigonometrical calculations nor logarithms will be wanted; a simple multiplication of two numbers will be sufficient. Combined with a small trigonometrical calculation they will give both conversions with nearly the same accuracy as can be obtained with logarithms having five figures of decimals.

Denoting the right ascension and declination of a heavenly body by $\alpha$ and $\delta$, the longitude and latitude by $\lambda$ and $\beta$, the obliquity of the elliptic by $\varepsilon$, the well-known formulæ for effecting both these mutual conversions are as follows:

$$
\begin{aligned}
\sin \beta & =\cos \varepsilon \sin \delta-\sin \varepsilon \cos \delta \sin \alpha \\
\cos \beta \sin \lambda & =\sin \varepsilon \sin \delta+\cos \varepsilon \cos \delta \sin \alpha \\
\cos \beta \cos \lambda & =\cos \alpha \cos \delta
\end{aligned}
$$

- From his Astronom. Jahrbuch, 1831, p. 281.-The explanation of the tables for converting geocentric longitude and latitude into right ascension and declination, and vice versâ, is here given, but not the tables, agreeably to the intention we have always had in giving these translations, which was to render the work of Encke useful to such as are not acquainted with the German language. This paper concludes the list of those from Encke's Ephemeris for 1831, of which we have given whatever was capable of being translated, as we formerly did of that for 1830.--Evir. 


$$
\begin{aligned}
\sin \delta & =\sin \varepsilon \cos \beta \sin \lambda+\sin \beta \cos \varepsilon \\
\cos \delta \sin \alpha & =\cos \varepsilon \cos \beta \sin \lambda-\sin \beta \sin \varepsilon \\
\cos \delta \cos \alpha & =\cos \beta \cos \lambda
\end{aligned}
$$

They are general through the whole circumference of the circle, inasmuch as, by the nature of the case, $\cos \alpha$ and $\cos \lambda$ have always the same signs, or as $\alpha$ and $\lambda$ are always at the same time between $90^{\circ}$ and $270^{\circ}$, or $270^{\circ}$ and $90^{\circ}$.

For facilitating the logarithmic calculation it is usual to introduce an auxiliary angle, by means of which the two parts on one side are changed into one only. For (1) put:

$$
\begin{aligned}
\sin \delta & =M \sin N \\
\cos \delta \sin \alpha & =M \cos N \\
\sin \beta & =M \sin (N-\varepsilon) \\
\cos \beta \sin \lambda & =M \cos (N-\varepsilon) \\
\cos \beta \cos \lambda & =\cos \delta \cos \alpha
\end{aligned}
$$

hence

The value of $M$ from the two first anxiliary formulæ being substituted in the latter three; viz. $M=\frac{\cos \delta \sin \alpha}{\cos N}$ we obtain

$$
\begin{aligned}
\operatorname{tang} N & =\frac{\operatorname{tang} \delta}{\sin \alpha} \\
\operatorname{tang} \lambda & =\frac{\cos (N-s)}{\cos N} \operatorname{tang} \alpha \\
\operatorname{tang} \beta & =\operatorname{tang}(N-\varepsilon) \sin \lambda
\end{aligned}
$$

to which may be added as a check on the calculation

$$
\frac{\cos (N-s)}{\cos N}=\frac{\cos \beta \sin \lambda}{\cos \delta \sin \alpha}
$$

These formulæ give every thing by means of tangents, consequently in the most accurate manner; and (with due regard to the remark above made) without any ambiguity; the geometrical signification of $\mathrm{M}$ and $\mathrm{N}$ will be easily found. For a single calculation they are beyond doubt the most convenient; but they do not admit of a table of single entry even for constant $\varepsilon$, and have besides the small disadvantage that for converting at the same time several places with regular differences, the auxiliary angle $\mathrm{N}$ is, in the vicinity of $0^{\circ}$ and $180^{\circ}$, more irregular than the given principal quantities.

For the system (2) we have, in like manner:

$$
\begin{aligned}
\operatorname{tang} N^{\prime} & =\frac{\operatorname{tang} \beta}{\sin \lambda} \\
\operatorname{tang} \alpha & =\frac{\cos N^{\prime}+2}{\cos N^{\prime}} \operatorname{tang} \lambda \\
\operatorname{tang} \delta & =\operatorname{tang}\left(N^{\prime}+\varepsilon\right) \sin \alpha \\
\frac{\cos N^{\prime}+1}{\cos N} & =\frac{\cos \delta \sin \alpha}{\cos \beta \sin \lambda} \\
2 & U^{2}
\end{aligned}
$$


The formula (1) and (2) may be rendered convenient for logarithmic calculation in another way, by assuming for each of the two systems a particular case as a foundation.

Designating in (1) by $\lambda^{\prime}$ and $\delta^{\prime}$ the longitude and declination of a point whose right ascension $=\alpha, \alpha$, and latitude $=0$, we have $0=\cos \varepsilon \sin \delta^{\prime}-\sin \varepsilon \cos \delta^{\prime} \sin \alpha$ $\sin \lambda^{\prime}=\sin \varepsilon \sin \delta^{\prime}+\cos \varepsilon \cos \delta^{\prime} \sin \alpha$

whence

$$
\cos \lambda^{\prime}=\cos \alpha \cos \delta^{\prime}
$$

$$
\begin{aligned}
& \sin \lambda^{\prime} \sin \varepsilon=\sin \delta^{\prime} \\
& \sin \lambda^{\prime} \cos \varepsilon=\cos \delta^{\prime} \sin \alpha \\
& \cos \lambda^{\prime}=\cos \delta^{\prime} \cos \alpha
\end{aligned}
$$

Combining (1) and (3) by multiplying with $\cos \delta$ and $\cos \delta^{\prime}$, as also with $\cos \lambda^{\prime}$ and $\sin \lambda^{\prime}$, we have

$$
\sin \beta=\frac{\cos \varepsilon}{\cos \delta^{\prime}} \sin \left(\delta-\delta^{\prime}\right)
$$

$\cos \beta \sin \left(\lambda-\lambda^{\prime}\right)=\sin \varepsilon \cos \alpha \sin \left(\delta-\delta^{\prime}\right)$

$\cos \beta \cos \left(\lambda-\lambda^{\prime}\right)=\cos \delta \cos \delta^{\prime} \cos \alpha^{2}+\sin \varepsilon^{2} \sin \delta \sin \delta^{\prime}$ $+\cos \varepsilon^{2} \sin \alpha^{2} \cos \delta \cos \delta^{\prime}$

$+\sin \varepsilon \cos \varepsilon \sin \alpha \sin \left(\delta+\delta^{\prime}\right)$

where the latter formula may also be written thus:

$$
\begin{aligned}
\cos \beta \cos \left(\lambda-\lambda^{\prime}\right)= & \cos \left(\delta-\delta^{\prime}\right) \\
& -\{\cos \varepsilon \sin \delta-\sin \varepsilon \cos \delta \sin \alpha\} \times \\
& \left\{\cos \varepsilon \sin \delta^{\prime}-\sin \varepsilon \cos \delta^{\prime} \sin \alpha\right\}
\end{aligned}
$$

and as by the first equation of (3) the latter part is always $=0$, we have

$$
\begin{gathered}
\sin \beta=\frac{\cos \varepsilon}{\cos \delta} \cdot \sin \left(\delta-\delta^{\prime}\right) \\
\cos \beta \sin \left(\lambda-\lambda^{\prime}\right)=\sin \varepsilon \cos \alpha \sin \left(\delta-\delta^{\prime}\right) \\
\cos \beta \cos \left(\lambda-\lambda^{\prime}\right)=\cos \left(\delta-\delta^{\prime}\right)
\end{gathered}
$$

These three equations squared, give

$$
1=\left\{\frac{\cos \varepsilon^{2}}{\cos \delta^{\prime 2}}+\sin \varepsilon^{2} \cos \alpha^{2}\right\} \sin \left(\delta-\delta^{\prime}\right)^{2}+\cos \left(\delta-\delta^{\prime}\right)^{2}
$$

It will, therefore, be allowed to put

$$
\frac{\cos s}{\cos \gamma^{\prime}}=\sin \gamma, \quad \sin \varepsilon \cos \alpha=\cos \gamma
$$

where on account of the first equation of (5) $\gamma$ must always be assumed less than $180^{\circ}$. The calculation is thus reduced to these six equations:

$$
\begin{aligned}
\operatorname{tang} \lambda^{\prime} & =\operatorname{tang} \alpha \sec \varepsilon \\
\operatorname{tang} \delta^{\prime} & =\sin \alpha \operatorname{tang} \varepsilon \\
\cos \gamma & =\cos \alpha \sin \varepsilon \\
\sin \beta & =\sin \gamma \sin \left(\delta-\delta^{\prime}\right) \\
\cos \beta \sin \left(\lambda-\lambda^{\prime}\right) & =\cos \gamma \sin \left(\delta-\delta^{\prime}\right) \\
\cos \beta \cos \left(\lambda-\lambda^{\prime}\right) & =\cos \left(\delta-\delta^{\prime}\right)
\end{aligned}
$$

in which $\cos \lambda^{\prime}$ and $\cos \alpha$ must always be taken with equal 
signs. In these equations $\lambda^{\prime}, \delta^{\prime}$ and $\gamma$ for a constant $\varepsilon$ are functions of $\alpha$ only. If, therefore, $\lambda^{\prime}, \delta^{\prime}, \sin \gamma, \cos \gamma$, be reduced into tables with single entry, the argument being $\alpha$, it will only be required to calculate,

$$
\begin{aligned}
\operatorname{tang} p & =\operatorname{tang}\left(\delta-\delta^{\prime}\right) \cos \gamma \\
\operatorname{tang} \beta & =\operatorname{tang}\left(\delta-\delta^{\prime}\right) \sin \gamma \cos p \\
\lambda & =\lambda^{\prime}+p .
\end{aligned}
$$

The geometrical signification of these auxiliary quantities will be found without trouble.

Exactly in the same manner we have for system (2),

$$
\begin{array}{r}
\operatorname{tang} \alpha^{\prime}=\operatorname{tang} \lambda \sec \varepsilon \\
\operatorname{tang} \beta^{\prime}=\sin \lambda \operatorname{tang} \varepsilon \\
\cos \gamma^{\prime}=\cos \lambda \sin \varepsilon \\
\sin \delta=\sin \gamma^{\prime} \sin \left(\beta+\beta^{\prime}\right)
\end{array}
$$$$
\cos \delta \sin \left(\alpha^{\prime}-\alpha\right)=\cos \gamma^{\prime} \sin \left(\beta+\beta^{\prime}\right)
$$$$
\cos \delta \cos \left(\alpha^{\prime}-\alpha\right)=\cos \left(\beta+\beta^{\prime}\right)
$$

If, therefore, the following quantities are reduced to tables whose argument $k$ :

$$
\begin{aligned}
\operatorname{tang} \mathbf{A} & =\operatorname{tang} k \sec \varepsilon \\
\operatorname{tang} \mathbf{B} & =\sin k \operatorname{tang} \varepsilon \\
a & =\cos k \sin \varepsilon \\
b & =\frac{\cos \varepsilon}{\cos B}
\end{aligned}
$$

we shall find for

$$
\begin{aligned}
& \operatorname{tang} p=a \operatorname{tang}(\delta-\mathrm{B}) \quad \lambda=\mathbf{A}+p \\
& \operatorname{tang} \beta=b \operatorname{tang}(\delta-\mathrm{B}) \cos p
\end{aligned}
$$

and for

$$
k=\lambda
$$

$$
\begin{aligned}
& \operatorname{tang} q=a \operatorname{tang}(\beta+\mathrm{B}) \quad \alpha=\mathrm{A}-q \\
& \operatorname{tang} \delta=b \operatorname{tang}(\beta+\mathrm{B}) \cos q
\end{aligned}
$$

With respect to the purpose for which the tables are here intended, viz. converting the geocentric $\alpha$ and $\delta$ into $\lambda$ and $\beta$, for planets or such heavenly bodies as are within the zodiac, or for which $\beta< \pm 10^{\circ}$, it will be sufficient, if an approximation is only required, to put: $\quad \beta=b(\delta-\mathrm{B})$

$$
\lambda=\mathrm{A}+a(\delta-\mathrm{B}) \sec \beta
$$

where even the factor sec $\beta$ may in most cases be neglected, because its value is only,

\begin{tabular}{|r|c|r|c|}
\hline \multicolumn{1}{|c|}{$\beta$} & $\sec \beta$ & $\beta$ & $\sec \beta$ \\
\hline $\pm 0^{\circ}$ & 1.000 & $\pm 6^{\circ}$ & 1.006 \\
1 & 1.000 & 7 & 1.008 \\
2 & 1.001 & 8 & 1.010 \\
3 & 1.001 & 9 & 1.012 \\
4 & 1.002 & 10 & 1.015 \\
5 & 1.004 & & \\
\hline
\end{tabular}


334 Mr. De la Beche on the Geographical Distribution of Organic

The errors arising from neglecting this factor will always be within narrow limits. For the rigorous value of $\beta$ we have $\sin \beta=b \sin \left(\delta-\delta^{\prime}\right)$ and by developing the series:

$$
\beta=b\left(\delta-\delta^{\prime}\right)-\frac{1}{6} \sin \beta^{3} \operatorname{cotang} \gamma^{2}-\frac{3}{40} \sin \beta^{5} \operatorname{cotang} \gamma^{2} \frac{1+\sin \gamma^{2}}{\sin \gamma^{2}} \ldots
$$

where the last term when a maximum for $\beta=10^{\circ}$ does not amount to a second; the second, when a maximum, for

$$
\begin{aligned}
\beta & =5^{\circ} \ldots \ldots \ldots \ldots . .44^{\prime \prime} \\
& =10 \ldots \ldots \ldots . .34
\end{aligned}
$$

'The error for $\lambda$ would be somewhat greater: here the development of the series is

$$
\lambda=\mathbf{A}+a\left(\delta-\delta^{\prime}\right) \sec \beta-\frac{1}{6} \frac{\operatorname{tang} \beta^{3}}{\operatorname{tang} \gamma} \ldots .
$$

For $\beta=5^{\circ}$ the error is about $9^{\prime \prime}$; for $\beta=10^{\circ}$ about $82^{\prime \prime}$ when a maximum. For the following tables, $\varepsilon$ has been assumed at $23^{\circ} 27^{\prime} 30^{\prime \prime}$.

As an example, let the longitude and latitude of Mars 1831, January, be required. The Ephemeris gives

$$
\alpha=0^{\mathrm{h}} 58^{\prime} 33^{\prime \prime} \cdot 65 \quad \delta=+6^{\circ} 42^{\prime} 0^{\prime \prime} \cdot 0 \text {. }
$$

By these quantities we find from the table,

$$
\begin{array}{cc}
\mathrm{A}=15^{\circ} 53^{\prime} \cdot 7 & \mathrm{~B}=+6^{\circ} 15^{\prime} \cdot 5 \\
a=+03852 & b=0.9228 \\
\varepsilon-\mathrm{B}=+26 \cdot 5 & \beta=+0^{\circ} 24^{\prime} \cdot 5 \\
a(\delta-\mathrm{B})=+10 \cdot 2 & \lambda=16^{\circ} 3^{\prime \cdot 9}
\end{array}
$$

The rigorous calculation would have given $\beta=0^{\circ} 24^{\prime} 25^{\prime \prime}$ and $\lambda=16^{\circ} 3^{\prime} 54^{\prime \prime}$.

XLIX. Notes on the Geographical Distribution of Organic Remains contained in the Oolitic Series of the Great London and Paris Basin, and in the same Series of the South of France. By Henry T. De la Beche, F.R.S. \& c.

[Continued from page 268.]

I $\mathrm{T}$ cannot have escaped the reader's observation, that many fossils are common to several parts of the oolitic series; - to show the various beds in which the same organic remains may occur, and at the same time to convey an idea of the organic contents of the oolitic series in general, I have formed the following table from the authorities already mentioned, and from others that will be noticed in their places. Such a table must necessarily be very defective, and can only answer temporary purposes; but it is hoped that it may be useful. 